\title{
Zeros of the Jones polynomial are dense in the complex plane
}

\author{
Xian'an Jin* Fuji Zhang \\ School of Mathematical Sciences, Xiamen University, \\ Xiamen, Fujian 361005, P.R.China \\ xajin@xmu.edu.cn fjzhang@xmu.edu.cn \\ Fengming Dong Eng Guan Tay
}

Mathematics and Mathematics Education, National Institute of Education

Nanyang Technological University, Singapore 637616, Singapore

fengming.dong@nie.edu.sg engguan.tay@nie.edu.sg

Submitted: Apr 13, 2010; Accepted: Jun 28, 2010; Published: Jul 10, 2010

Mathematics Subject Classification: 05C10,05C22,05C31,57M15,57M25,57M27,82B20

\begin{abstract}
In this paper, we present a formula for computing the Tutte polynomial of the signed graph formed from a labeled graph by edge replacements in terms of the chain polynomial of the labeled graph. Then we define a family of 'ring of tangles' links and consider zeros of their Jones polynomials. By applying the formula obtained, Beraha-Kahane-Weiss's theorem and Sokal's lemma, we prove that zeros of Jones polynomials of (pretzel) links are dense in the whole complex plane.
\end{abstract}

\section{Introduction}

Let $L$ be an oriented link, and $D$ be a diagram of $L$. Let $V_{L}(t)$ be the Jones polynomial [1] of $L$. The writhe $w(D)$ of $D$ is defined to be the sum of signs of the crossings of $L$. Let $[D]$ be the one-variable Kauffman bracket polynomial [2] of $D$ in $A$ (with the orientation of $D$ coming from $L$ ignored). Let

$$
f_{L}(A)=\left(-A^{3}\right)^{-w(D)}[D] .
$$

Then [2]

$$
V_{L}(t)=f_{L}\left(t^{-1 / 4}\right)
$$

\footnotetext{
${ }^{*}$ Corresponding author.
} 
It is well known that there is a one-to-one correspondence between link diagrams and signed plane graphs via the medial construction [3]. Based on this correspondence, in [3] Kauffman converted the Kauffman bracket polynomial to the Tutte polynomial of signed graphs, which are not necessarily planar. Let $G$ be a signed graph. We shall denote by $Q[G]=Q[G](A, B, d) \in \mathbb{Z}[A, B, d]$ the Tutte polynomial of $G$. To analyze zeros of Jones polynomials, it suffices for us to consider the Tutte polynomials of signed graphs.

There have been some works on zeros of Jones polynomials [4]-[9]. For example, the distribution of zeros of Jones polynomials for prime knots with small crossing number has been depicted in [4] and [5]. See Fig. 1 for an example. Looking at these figures, one may be tempted to conclude that zeros of Jones polynomials do not exist in some regions, for example, a small circle region around $z=1$ and a large area in the left half complex plane. But in this paper by considering zeros of pretzel links we shall show that, on the contrary, zeros of Jones polynomial of knots are dense in the whole complex plane. We point out that Sokal proved that chromatic roots are dense in the whole complex plane [10], and Zhang and Chen proved eigenvalues of digraphs are dense in the whole complex plane [11].

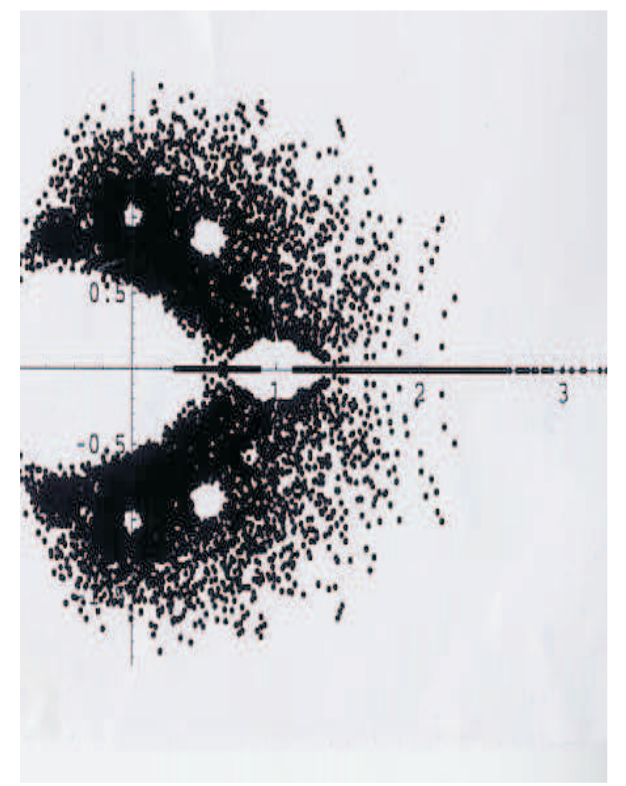

Fig. 1: Zeros of 1288 prime alternating knots with crossing number 12 [4].

This paper contains two parts. The first part is on a formula of computing the Tutte polynomial of signed graphs formed by edge replacements via the chain polynomial [12]. This result generalizes our previous results in [13]. It is worth noting that there are two closely related results in [14] and [15]. In the second part, we consider the Tutte polynomial of 'ring of tangles' links which include the well known pretzel links. By applying BerahaKahane-Weiss's Theorem and Sokal's lemma, we prove that zeros of the Jones polynomial of pretzel knots are dense in the complex plane. 


\section{Tutte polynomials of signed graphs formed by edge replace- ments}

The chain polynomial was introduced by Read and Whitehead in [12] for studying the chromatic polynomials of homeomorphic graphs. It is defined on labeled graphs, i.e. graphs whose edges have been labeled with elements of a commutative ring with unity. Although in a labeled graph different edges can receive the same label, we usually denote

the edges by the labels associated with them. The chain polynomial $C h[G]$ of a labeled graph $G$ is defined as

$$
C h[G]=\sum_{Y \subset E} F_{G-Y}(1-w) \prod_{a \in Y} a
$$

where the sum is over all subsets of the edge set $E$ of $G, F_{G-Y}(1-w)$ denotes the flow polynomial in $q=1-w$ of $G-Y$, the graph obtained from $G$ by deleting the edges in $Y$, and $\prod_{a \in Y}$ a denotes the product of labels in $Y$.

The following lemma was given implicitly in [12] and explicitly in [15]. It can be viewed as an alternative definition of the chain polynomial.

Lemma 2.1 The chain polynomial satisfies the following recursive rules:

(1) If $G$ is edgeless, then

$$
C h[G]=1
$$

(2) Otherwise, suppose a is an edge of $G$, we have:

(a) If a is a loop, then

$$
C h[G]=(a-w) C h[G-a] .
$$

(b) If a is not a loop, then

$$
C h[G]=(a-1) C h[G-a]+C h[G / a] .
$$

Example 2.2 (1) Let $\Theta_{p}$ be the labeled graph consisting of two vertices connected by $p$ parallel edges $a_{1}, a_{2}, \cdots, a_{p}$. Then [7]

$$
C h\left[\Theta_{p}\right]=\frac{1}{1-w}\left[\prod_{i=1}^{p}\left(a_{i}-w\right)-w \prod_{i=1}^{p}\left(a_{i}-1\right)\right] .
$$

(2) Let $B_{p}$ be a labeled "bouquet of $p$ circles", i.e. a labeled graph with one vertex and $p$ loops $a_{1}, a_{2}, \cdots, a_{p}$. By Lemma 2.1, we obtain

$$
C h\left[B_{p}\right]=\prod_{i=1}^{p}\left(a_{i}-w\right)
$$


Definition 2.3 Let $G$ be a connected labeled graph. We define $\hat{G}$ to be the signed graph obtained from $G$ by replacing each edge $a=u w$ of $G$ by a connected signed graph $H_{a}$ with two attached vertices $u$ and $w$ that has only the vertices $u$ and $w$ in common with $\widehat{G-a}$.

Now we shall establish a relation between the Tutte polynomial of $\hat{G}$ and the chain polynomial of $G$. The following two splitting lemmas on Tutte polynomials of signed graphs will be used in proving Theorem 2.6.

Lemma $2.4[3]$

(1) Let $G_{1} \cup G_{2}$ be the disjoint union of two signed graphs $G_{1}$ and $G_{2}$. Then

$$
Q\left[G_{1} \cup G_{2}\right]=d Q\left[G_{1}\right] Q\left[G_{2}\right] .
$$

(2) Let $G_{1} \cdot G_{2}$ be the union of two signed graphs $G_{1}$ and $G_{2}$ having only one common vertex. Then

$$
Q\left[G_{1} \cdot G_{2}\right]=Q\left[G_{1}\right] Q\left[G_{2}\right]
$$

Lemma 2.5 [16] Let $G$ be the union of two signed graphs $G_{1}$ and $G_{2}$ having only two common vertices $u_{1}$ and $u_{2}$. Let $H_{1}$ and $H_{2}$ be signed graphs obtained from $G_{1}$ and $G_{2}$, respectively, by identifying $u_{1}$ and $u_{2}$. Then

$$
Q[G]=\frac{1}{d^{2}-1}\left\{d Q\left[H_{1}\right] Q\left[H_{2}\right]+d Q\left[G_{1}\right] Q\left[G_{2}\right]-Q\left[H_{1}\right] Q\left[G_{2}\right]-Q\left[G_{1}\right] Q\left[H_{2}\right]\right\} .
$$

Let $H_{a}^{\prime}$ be the graph obtained from $H_{a}$ by identifying $u$ and $w$, the two attached vertices of $H_{a}$. Let

$$
\begin{aligned}
& \alpha_{a}=\alpha\left[H_{a}\right]=\frac{1}{d^{2}-1}\left(d Q\left[H_{a}\right]-Q\left[H_{a}^{\prime}\right]\right), \\
& \beta_{a}=\beta\left[H_{a}\right]=\frac{1}{d^{2}-1}\left(d Q\left[H_{a}^{\prime}\right]-Q\left[H_{a}\right]\right), \\
& \gamma_{a}=\gamma\left[H_{a}\right]=1+d \frac{\alpha\left[H_{a}\right]}{\beta\left[H_{a}\right]}
\end{aligned}
$$

Theorem 2.6 Let $G$ be a connected labeled graph, and $\hat{G}$ be the signed graph obtained from $G$ by replacing the edge a by a connected signed graph $H_{a}$ for every edge $a$ in $G$. If we replace $w$ by $1-d^{2}$, and replace a by $\gamma_{a}$ for every label a in $C h(G)$, then we have

$$
Q[\hat{G}]=\frac{\prod_{a \in E(G)} \beta_{a}}{d^{q(G)-p(G)+1}} C h[G],
$$

where $p(G)$ and $q(G)$ are the numbers of vertices and edges of $G$, respectively. 
Proof. By solving Eqs. (10) and (11), we obtain

$$
Q\left[H_{a}^{\prime}\right]=\alpha_{a}+d \beta_{a} .
$$

When the edge $a$ is a loop of $G$, by Lemma 2.4 (2) and Eq. (14), we have

$$
\begin{aligned}
Q[\hat{G}] & =Q\left[H_{a}^{\prime}\right] Q[\widehat{G-a}] \\
& =\left(\alpha_{a}+d \beta_{a}\right) Q[\widehat{G-a}] .
\end{aligned}
$$

When the edge $a$ is not a loop, by Lemma 2.5 , we have

$$
\begin{aligned}
Q[\hat{G}] & =\frac{1}{d^{2}-1}\left\{d Q[\widehat{G / a}] Q\left[H_{a}^{\prime}\right]+d Q[\widehat{G-a}] Q\left[H_{a}\right]-Q[\widehat{G / a}] Q\left[H_{a}\right]-Q\left[H_{a}^{\prime}\right] Q[\widehat{G-a}]\right\} \\
& =\frac{1}{d^{2}-1}\left\{Q[\widehat{G-a}]\left(d Q\left[H_{a}\right]-Q\left[H_{a}^{\prime}\right]\right)+Q[\widehat{G / a}]\left(d Q\left[H_{a}^{\prime}\right]-Q\left[H_{a}\right]\right)\right\} \\
& =\alpha_{a} Q[\widehat{G-a}]+\beta_{a} Q[\widehat{G / a}] .
\end{aligned}
$$

Now let

$$
T[G]=\frac{d^{q(G)-p(G)+1}}{\prod_{a \in E(G)} \beta_{a}} Q[\hat{G}] .
$$

If $G$ is an edgeless graph with $n$ vertices, then

$$
T\left[E_{n}\right]=d^{-n+1} d^{n-1}=1 .
$$

Otherwise, suppose that $a$ is an edge of $G$.

(1) If $a$ is a loop of $G$, by Eq. (15), we have

$$
\begin{aligned}
T[G] & =\frac{d^{q(G)-p(G)+1}}{\prod_{b \in E(G)} \beta_{b}}\left(\alpha_{a}+d \beta_{a}\right) Q[\widehat{G-a}] \\
& =\frac{d^{q(G-a)+1-p(G-a)+1}}{\beta_{a} \prod_{b \in E(G-a)} \beta_{b}}\left(\alpha_{a}+d \beta_{a}\right) Q[\widehat{G-a}] \\
& =d\left(d+\frac{\alpha_{a}}{\beta_{a}}\right) T[G-a] .
\end{aligned}
$$

(2) If $a$ is not a loop, by Eq. (16), we have

$$
\begin{aligned}
T[G] & =\frac{d^{q(G)-p(G)+1}}{\prod_{b \in E(G)} \beta_{b}} Q[\hat{G}] \\
& =\frac{d^{q(G)-p(G)+1}}{\prod_{b \in E(G)} \beta_{b}}\left(\alpha_{a} Q[\widehat{G-a}]+\beta_{a} Q[\widehat{G / a}]\right) \\
& =\frac{d^{q(G-a)+1-p(G-a)+1}}{\beta_{a} \prod_{b \in E(G-a)} \beta_{b}} \alpha_{a} Q[\widehat{G-a}]+\frac{d^{q(G / a)+1-p(G / a)-1+1}}{\beta_{a} \prod_{b \in E(G-a)} \beta_{b}} \beta_{a} Q[\widehat{G / a}] \\
& =d \frac{\alpha_{a}}{\beta_{a}} T[G-a]+T[G / a] .
\end{aligned}
$$


Comparing the coefficients of Eqs. (17)-(19) with those of (2)-(4) in Lemma 2.1, we know that $T[G]=\left.C h[G]\right|_{w=1-d^{2}, a=\gamma_{a}}$. Theorem 2.6 follows directly.

Hereafter, we always suppose that $B=A^{-1}, d=-A^{2}-A^{-2}$. Let $X=A+B d=-A^{-3}$. Let $H_{a}=P_{n}^{+}$, a positive path with length $n$. Then $Q\left[P_{n}^{+}\right]=X^{n}$. It is not difficult to obtain

$$
Q\left[C_{n}^{+}\right]=\left(X^{n}-A^{n}\right) / d+d A^{n} .
$$

Thus,

$$
\begin{aligned}
\alpha\left[P_{n}^{+}\right] & =\left(X^{n}-A^{n}\right) / d, \\
\beta\left[P_{n}^{+}\right] & =A^{n}, \\
\gamma\left[P_{n}^{+}\right] & =(X / A)^{n}=\left(-A^{-4}\right)^{n} .
\end{aligned}
$$

Hence, we have

Corollary 2.7 [13] Let $G$ be a connected labeled graph. Let $G_{c}$ be the signed graph obtained from $G$ by replacing each edge a by a positive path with length $n_{a}$. In $C h[G]$, if we replace $w$ by $1-d^{2}$, and replace a by $\left(-A^{-4}\right)^{n_{a}}$ for every label a, then we have

$$
Q\left[G_{c}\right]=\frac{A^{\sum_{a \in E(G)} n_{a}}}{d^{q(G)-p(G)+1}} C h[G],
$$

where $p(G)$ and $q(G)$ are the numbers of vertices and edges of $G$, respectively.

Example 2.8 (1) The generalized theta graph $\Theta_{s_{1}, \cdots, s_{p}}$ consists of end-vertices $x, y$ connected by $p$ internally disjoint positive paths of lengths $s_{1}, \cdots, s_{p}$. By Eq. (5) and Corollary 2.7, we obtain

$$
Q\left[\Theta_{s_{1}, \cdots, s_{p}}\right]=\frac{A^{\sum s_{i}}}{d^{p+1}}\left[\prod_{i=1}^{p}\left(\left(-A^{-4}\right)^{s_{i}}+d^{2}-1\right)+\left(d^{2}-1\right) \prod_{i=1}^{p}\left(\left(-A^{-4}\right)^{s_{i}}-1\right)\right] .
$$

Note that the formula is essentially the same as the formula for Kaufman bracket polynomial of pretzel links in [17].

(2) Let $B_{s_{1}, \cdots, s_{p}}$ be the graph consisting of one vertex incident with $p$ internally disjoint positive cycles of lengths $s_{1}, \cdots, s_{p}$. By Eq. (6) and Corollary 2.7, we obtain

$$
Q\left[B_{s_{1}, \cdots, s_{p}}\right]=\frac{A^{\sum s_{i}}}{d^{p}} \prod_{i=1}^{p}\left(\left(-A^{-4}\right)^{s_{i}}+d^{2}-1\right) .
$$

By Example 2.8, we obtain

$$
\begin{aligned}
& \alpha\left[\Theta_{s_{1}, \cdots, s_{p}}\right]=\frac{A^{\sum s_{i}}}{d^{p}} \prod_{i=1}^{p}\left(\left(-A^{-4}\right)^{s_{i}}-1\right), \\
& \beta\left[\Theta_{s_{1}, \cdots, s_{p}}\right]=\frac{A^{\sum s_{i}}}{d^{p+1}}\left[\prod_{i=1}^{p}\left(\left(-A^{-4}\right)^{s_{i}}+d^{2}-1\right)-\prod_{i=1}^{p}\left(\left(-A^{-4}\right)^{s_{i}}-1\right)\right] .
\end{aligned}
$$




\section{Zeros of Jones polynomials are dense in the whole complex plane}

Suppose that $\left\{f_{n}(x) \mid n=1,2, \cdots\right\}$ is a family of polynomials. A complex number $z$ is said to be the limit of zeros of $\left\{f_{n}(x)\right\}$ if either $f_{n}(z)=0$ for all sufficiently large $n$ or $z$ is a limit point of the set $\Re\left(\left\{f_{n}(x)\right\}\right)$, where $\Re\left(\left\{f_{n}(x)\right\}\right)$ is the union of the zeros of the $f_{n}(x)^{\prime} s$. In [18], Beraha, Kahane and Weiss proved the following theorem.

Theorem 3.1 If $\left\{f_{n}(x)\right\}$ is a family of polynomials such that

$$
f_{n}(x)=\alpha_{1}(x) \lambda_{1}(x)^{n}+\alpha_{2}(x) \lambda_{2}(x)^{n}+\cdots+\alpha_{l}(x) \lambda_{l}(x)^{n},
$$

where the $\alpha_{i}(x)$ and $\lambda_{i}(x)$ are fixed non-zero polynomials, such that no pair $i \neq j$ has $\lambda_{i}(x) \equiv \omega \lambda_{j}(x)$ for some complex number $\omega$ of unit modulus, then $z$ is a limit of zeros of $\left\{f_{n}(x)\right\}$ if and only if

(1) two or more of the $\lambda_{i}(z)$ are of equal modulus, and strictly greater in modulus than the others; or

(2) for some $j$, the modulus of $\lambda_{j}(z)$ is strictly greater than those of the others, and $\alpha_{j}(z)=0$.

Now we define a family of 'ring of tangles' links. Let $H_{i}$ be a signed plane graph and $u_{i}, v_{i}$ be two distinct vertices of $H_{i}$ lying in the boundary of the unbounded face for $i=1,2, \cdots, n$. Let $C\left(H_{1}, H_{2}, \cdots, H_{n}\right)$ be the signed plane graph obtained by identifying $u_{i}$ with $v_{i+1}$ for each $i=1,2, \cdots, n-1$, and identifying $u_{n}$ with $v_{1}$. We denote by $L\left(T_{1}, T_{2}, \cdots, T_{n}\right)$ the link diagram corresponding to $C\left(H_{1}, H_{2}, \cdots, H_{n}\right)$ with the tangle $T_{i}$ corresponding to $H_{i}$ for $i=1,2, \cdots, n$, and call it a 'ring of tangles' link. In particular, if $H_{1}=H_{2}=\cdots=H_{n}=H$, we denote $C\left(H_{1}, H_{2}, \cdots, H_{n}\right)$ by $C^{n}(H)$ for simplicity.

Lemma 3.2 Let $L^{n}(T)$ be the link diagram corresponding to $C^{n}(H)$ with the tangle $T$ corresponding to the signed plane graph $H$. Let $Q(H)=\left.Q[H]\right|_{A=t^{-1 / 4}}$ and $\beta(H)=$ $\left.\beta[H]\right|_{A=t^{-1 / 4}}$. Then points satisfying the following equation

$$
|\beta(H)|=|Q(H)|
$$

are limits of zeros of Jones polynomials of $\left\{L^{n}(T) \mid n=1,2, \cdots\right\}$.

\section{Proof.}

(1) Let $C_{n}$ be the $n$-cycle, and its edges are all labeled with $a$. By using the definition of the chain polynomial, it is not difficult for us to obtain $C h\left[C_{n}\right]=a^{n}-w$. Replacing $a$ suitably by the signed plane graph $H$, we obtain $C^{n}(H)$. By Theorem 2.6, we have

$$
\begin{aligned}
Q\left[C^{n}(H)\right] & =\frac{\beta[H]^{n}}{d}\left(\gamma\left[H_{i}\right]^{n}-\left(1-d^{2}\right)\right) \\
& =\frac{d^{2}-1}{d} \beta[H]^{n}+\frac{1}{d}(\beta[H]+d \alpha[H])^{n} \\
& =\frac{d^{2}-1}{d} \beta[H]^{n}+\frac{1}{d} Q[H]^{n} .
\end{aligned}
$$


(2) By replacing $A$ by $t^{-1 / 4}$, we obtain

$$
V_{L^{n}(T)}(t) \doteq \frac{1}{t+1}\left\{\left(t^{2}+t+1\right) \beta(H)^{n}+t Q(H)^{n}\right\}
$$

where $\doteq$ denotes equality up to a factor $\pm t^{k / 2}$. Then by applying Beraha-KahaneWeiss's Theorem, we obtain the lemma.

The following Sokal's lemma [10] will play an important role in proving our main result.

Lemma 3.3 Let $F_{1}, F_{2}, G$ be analytic functions on a disc $|z|<R$ satisfying $|G(0)| \leqslant 1$ and $G$ not constant. Then, for each $\epsilon>0$, there exists $s_{0}<\infty$ such that for all integers $s \geqslant s_{0}$ the equation

$$
\left|1+F_{1}(z) G(z)^{s}\right|=\left|1+F_{2}(z) G(z)^{s}\right|
$$

has a solution in the disc $|z|<\epsilon$.

Lemma 3.4 Let $t_{0}$ be any complex number. For any real $\epsilon>0$, there is a signed plane graph $H$ such that Eq. (26) has a zero $t$ with $\left|t-t_{0}\right|<\epsilon$.

Proof. Let $I_{s}$ be the graph with two vertices $x$ and $y$, and $s$ parallel edges joining $x$ and $y$. Denote by $I_{s}^{+}$and $I_{s}^{-}$the two signed graphs obtained from $I_{s}$ by assigning its each edge a positive sign and a negative sign, respectively. By setting $p=s$ and $s_{1}=s_{2}=\cdots=s_{p}=1$ in Eqs. (23) and (24), we obtain

$$
\begin{aligned}
\alpha\left[I_{s}^{+}\right] & =A^{-s} \\
\beta\left[I_{s}^{+}\right] & =\frac{\left(-A^{3}\right)^{s}-A^{-s}}{-A^{2}-A^{-2}} .
\end{aligned}
$$

Thus we have

$$
Q\left[I_{s}^{+}\right]=\beta\left[I_{s}^{+}\right]+d \alpha\left[I_{s}^{+}\right]=\frac{1}{-A^{2}-A^{-2}}\left[\left(A^{4}+A^{-4}+1\right) A^{-s}+\left(-A^{3}\right)^{s}\right] .
$$

Then the equation

$$
\left|\beta\left[I_{s}^{+}\right]\right|=\left|Q\left[I_{s}^{+}\right]\right|
$$

is equivalent to

$$
\left|1+(-1)^{s-1} A^{-4 s}\right|=\left|1+(-1)^{s}\left(A^{4}+A^{-4}+1\right) A^{-4 s}\right| .
$$

Letting $A=t^{-1 / 4}$, this equation is transformed into

$$
\left|1-(-t)^{s}\right|=\left|1+\left(t+t^{-1}+1\right)(-t)^{s}\right| .
$$


Let $t_{0}$ be an any fixed complex number with $\left|t_{0}\right| \leqslant 1$ and $t_{0} \neq 0$. Setting $z=t-t_{0}$, Eq. (29) becomes

$$
\left|1-\left(-z-t_{0}\right)^{s}\right|=\left|1+\left(z+t_{0}+\left(z+t_{0}\right)^{-1}+1\right)\left(-z-t_{0}\right)^{s}\right| .
$$

By Sokal's Lemma $\left(F_{1}(z)=-1, F_{2}(z)=\left(z+t_{0}+\left(z+t_{0}\right)^{-1}+1\right), G(z)=\left(-z-t_{0}\right)\right)$, then for any $\epsilon>0$, there exists $s_{0}$ such that for any $s \geqslant s_{0}$, Eq. (30) has a zero $z$ satisfying $|z|<\epsilon$, i.e. Eq. (29) has a zero $t=z+t_{0}$ satisfying $\left|t-t_{0}\right|<\epsilon$.

For the special case that $t_{0}=0$, by the above result, there exists $s_{0}$ such that for any $s \geqslant s_{0}$, Eq. (29) has a zero $t$ satisfying $|t-\epsilon / 2|<\epsilon / 2$, implying that $|t|<\epsilon$.

Now consider $I_{s}^{-}$, in this case, Eq. (29) becomes

$$
\left|1-\left(-t^{-1}\right)^{s}\right|=\left|1+\left(t+t^{-1}+1\right)\left(-t^{-1}\right)^{s}\right| .
$$

If $\left|t_{0}\right| \geqslant 1$, by Sokal's Lemma, for any $\epsilon>0$, there exists $s_{0}$ such that for any $s \geqslant s_{0}$, Eq. (31) has a zero $t$ satisfying $\left|t-t_{0}\right|<\epsilon$.

Theorem 3.5 Zeros of Jones polynomials are dense in the whole complex plane.

Proof. Let $t_{0}$ be any complex number and $\epsilon$ any positive real number. By Lemma 3.4, there exists a signed plane graph $H$ such that Eq. (26) has a zero $t^{\prime}$ with $\left|t^{\prime}-t_{0}\right|<\epsilon / 2$. Then, by Lemma 3.2, there exists an integer $n>0$ such that $V_{L^{n}(T)}(t)$ has a zero $t$ with $\left|t-t^{\prime}\right|<\epsilon / 2$. Together, these mean that there exists a zero $t$ of some Jones polynomial with $\left|t-t_{0}\right|<\epsilon$.

Finally, we give two remarks on Theorem 3.5.

1. Note that $C^{n}\left(I_{s}^{+}\right)$and $C^{n}\left(I_{s}^{-}\right)$correspond to the pretzel link $P(\overbrace{s, s, \ldots, s}^{n})$ with $s>0$ and $s<0$. We actually prove that the zeros of Jones polynomials of pretzel links are dense in the whole complex plane.

2. Using Beraha-Kahane-Weiss's Theorem we can also prove that points of Eq. (26) are also limits of zeros of Jones polynomials of the link subfamily $\left\{L^{2 k+1}(T) \mid k=\right.$ $1,2, \cdots\}$. Note that when $n$ and $s$ are both odd numbers, $P(\overbrace{s, s, \ldots, s}^{n})$ is a knot. Hence, we can further prove that zeros of Jones polynomial of such pretzel knots are dense in the whole complex plane.

Acknowledgements This paper was partially finished during Xian'an Jin's visit at the National Institute of Education, Singapore, supported by NIE AcRf Funding (R1 5/06 DFM). Xian'an Jin and Fuji Zhang are also partially supported by NSFC Grant No. 10831001. 


\section{References}

[1] V.F.R. Jones, A polynomial invariant for knots via Von Neumann algebras, Bull. Am. Math. Soc. 12 (1985) 103-112.

[2] L.H. Kauffman, State models and the Jones polynomial, Topology 26 (1987) 395-407.

[3] L.H. Kauffman, A Tutte polynomial for signed graphs, Discrete Appl. Math. 25 (1989) 105-127.

[4] X.-S. Lin, Zeros of the Jones polynomial, http://math.ucr.edu/ xl/abs-jk.pdf.

[5] F.Y. Wu, J. Wang, Zeros of the Jones polynomial, Physica A 296 (2001) 483-494.

[6] S.-C. Chang, R. Shrock, Zeros of Jones polynomials for families of knots and links, Physica A 301 (2001) 196-218.

[7] X. Jin, F. Zhang, Zeros of the Jones polynomials for families of pretzel links, Physica A 328 (2003) 391-408.

[8] A. Champanerkar, L. Kofman, On the Mahler measure of Jones polynomials under twisting, Algebr. Geom. Topol. 5 (2005) 1-22.

[9] S. Jablan, L. Radovic, R. Sazdanovic, Tutte and Jones polynomial of link families, Advanced School and Conference on Knot Theory and its Applications to Physics and Biology, May 11-29, 2009 International Centre for Theoretical Physics Trieste, Italy. http://atlas-conferences.com/c/a/y/x/08.htm

[10] A.D. Sokal, Chromatic roots are dense in the whole complex plane, Comb. Probab. Comput. 13 (2004) 221-261.

[11] F. Zhang and Z. Chen, Limit points of eigenvalues of (di)graphs, Czech. Math. J. 56 (2006) 895-902.

[12] R.C. Read, E.G. Whitehead Jr., Chromatic polynomials of homeomorphism classes of graphs, Discrete. Math. 204 (1999) 337-356.

[13] X. Jin, F. Zhang, The Kauffman brackets for equivalence classes of links, Adv. in Appl. Math. 34 (2005) 47-64.

[14] D.R. Woodall, Tutte polynomial expansions for 2-separable graphs, Discrete Math. 247 (2002) 201-213.

[15] L. Traldi, Chain polynomials and Tutte polynomials, Discrete Math. 248 (2002) 279282.

[16] X. Jin, F. Zhang, On computing Kauffman bracket polynomial of Montesinos links, J. Knot Theory Ramifications, accepted.

[17] P.M.G. Manchón, On the Kauffman bracket of pretzel links, Marie Curie Fellowships Annals, Second Volume, 2002. http://www.mariecurie.org/annals/volume2/manchon.pdf.

[18] S. Beraha, J. Kahane, N.J. Weiss, Limits of zeros of recursively defined families of polynomials, Studies in Foundations and Combinatorics, Advances in Mathematics Supplementary Studies, 1 (1978) 212-232. 